\title{
The Influence of the Pinning Centres Dimensions on the Critical Current in High- $T_{\mathrm{c}}$ Superconductors
}

\begin{abstract}
J. Sosnowski
Electrotechnical Institute, Pożaryskiego 28, 04-703 Warsaw, Poland

An analysis of the influence of the pinning centres dimensions on the critical current and pinning forces of the high temperature oxide superconductors is presented. Individual interaction of the pinning centres with pancake type vortices is considered for the layered structure of the copper-oxide superconductors. The phenomenological model was elaborated in which the change of the system energy has been constructed taking into account the pinning interaction, Lorentz forces, and elastic energy of the vortex lattice. The resistivity criterion has been used for detection of the critical current. Especially important results have been obtained for the range of the pinning centres dimensions smaller than twice the value of the coherence length.
\end{abstract}

PACS numbers: 74.25.Qt, 74.72.--h

\section{Introduction}

The magnitude of the transport current in superconductors is strongly dependent on the existence of structural defects, which can act as the pinning centres. In the high temperature superconductors the coherence length describing the radius of the vortex is of the order of several nanometers, so the flux pinning takes place on the nanometer scale. The analysis of this problem is the subject of the present paper, with the emphasis directed especially on the determination of the influence of the pinning centres dimensions on the electromagnetic properties, such as critical current and pinning forces. 


\section{The model and results of calculations}

In the high temperature oxide superconductors the pancake vortices are generated in the perpendicular magnetic field. They are localized in various layers of the thickness $(l)$ and interact with individual pinning centres of nanoscale range. The analysis of this interaction has meaning both for the basic investigations as well as because it is a frequently appearing case when the superconductor is exposed to the heavy ions irradiation. Then the structural defects with the dimensions of the range of a few nanometers are created (columnar defect). Due to the pinning interaction the potential barrier $\Delta U$ arises, which is a function of the current [1], for a flat pinning centre given then by the following relation:

$$
\Delta U=\frac{\mu_{0} H_{\mathrm{c}}^{2}}{2} l \xi_{0}^{2}\left[\arcsin \left(-\frac{j}{j_{\mathrm{c}}}\right)+\frac{\pi}{2}-\frac{j}{j_{\mathrm{c}}} \sqrt{1-\left(-\frac{j}{j_{\mathrm{c}}}\right)^{2}}\right] .
$$

In Eq. (1) the parameter $j_{\mathrm{c}}$ appears having the meaning of the critical current density, because for $i=j / j_{\mathrm{c}}=1$ the potential barrier vanishes, while for a large flat pinning centre $j_{\mathrm{c}}$ is given by the following relation:

$$
j_{\mathrm{c}}=\frac{\mu_{0} H_{\mathrm{c}}^{2}}{\pi \xi_{0} B} \frac{S\left(1-S / a^{2}\right)}{a^{2}},
$$

$S$ is an area of the pinning centre and the last factor is connected with the filling of superconductor by the normal phase pinning centres. $H_{\mathrm{c}}$ is thermodynamic critical field, while $\xi_{0}$ is the coherence length. The symbol $a$ is the distance between pinning centres, which for an ordered arrangement of pinning centres is simply expressed by the pinning centre concentration. However for nanoscale dimensions of the pinning centres Eq. (1) should be modified. The scheme of the investigated geometry of the interacting nano-pinning centre in the form of thin slab with the vortex is shown in Fig. 1. In an initial state, the vortex captured on the depth equal to the coherence length $\xi_{0}$ describing the radius of the vortex core has been considered. During the movement of the vortex on the distance $x$ against the pinning centre, as indicated in Fig. 1, the normal part of energy of the

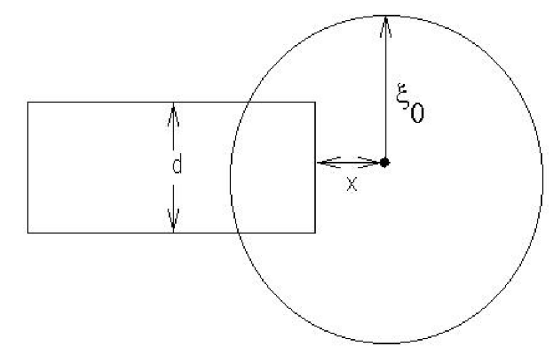

Fig. 1. The view of the vortex with the core of the radius equal to the coherence length $\left(\xi_{0}\right)$ captured on the pinning centre of the width $(d)$. 
superconductor with the captured vortex increases. The potential barrier which is additionally modified by the Lorentz forces [2] and elasticity energy arises. Finally the maximal potential barrier height $\Delta U_{1}$ for $d<2 \xi_{0}$ is given as:

$$
\begin{aligned}
\Delta U_{1} & =\frac{\mu_{0} H_{\mathrm{c}}^{2} l \xi^{2}}{2}\left[-\arcsin i+\arcsin \frac{d}{2 \xi_{0}}+\frac{d}{2 \xi_{0}} \sqrt{1-\left(\frac{d}{2 \xi_{0}}\right)^{2}}-i \sqrt{1-i^{2}}\right] \\
& +\alpha \xi_{0} \sqrt{1-i^{2}}\left(\sqrt{1-i^{2}}-2\right) .
\end{aligned}
$$

The elasticity energy of the vortex lattice has been taken into account in Eq. (3) in the first approximation of a small parameter $\alpha$ limit. The parameter $\alpha$ is the proportionality coefficient between the increase in the elasticity energy $U_{\mathrm{el}}$ and deflection $(x)$ of the vortex from an equilibrium position:

$$
U_{\mathrm{el}}=\alpha\left(\xi_{0}-x\right)^{2} .
$$

The capturing of vortices by the nano-pinning centre causes the deviation of the vortex from its equilibrium position in the regular vortex array, thus leading to an increase in the elastic energy of the magnetic structure of the vortex lattice. This effect is the function of the deflection of the individual vortex from its equilibrium position in the lattice as indicates Eq. (4). The influence of the elastic properties of the vortex lattice on the critical current density is shown in Fig. 2.

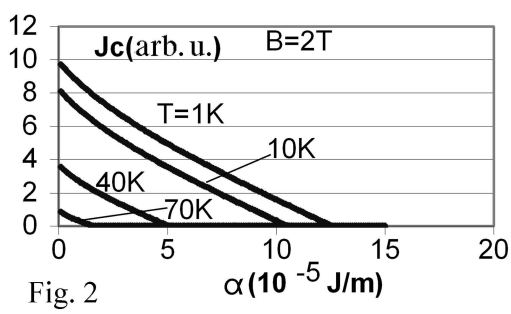

Fig. 2

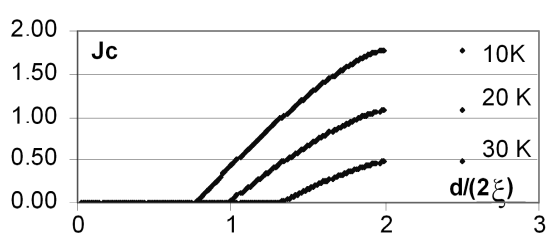

Fig. 3

Fig. 2. The influence of the elastic properties of the vortex lattice, expressed by the parameter $\alpha$ on the critical current density, versus temperature.

Fig. 3. The dependence of the critical current density in arbitrary units on the temperature versus dimensions of the pinning centres in the nanoscale range.

The potential barrier height given by Eq. (3) is a function of the pinning centre width $d$ as well as of the current density $j$. It indicates that the critical current determined by the condition $\Delta U_{1}=0$ obtained from Eq. (3) or more accurately, fulfilling the resistivity criterion from the flux creep equation is also a function of the pinning centre dimension. The results of the numerical calculations of the influence of the pinning centres dimensions on the critical current density is shown in Fig. 3 for three temperatures and it indicates that too small pinning centres of the nanoscale range are not sufficiently effective for the superconducting current flow. The following dependence of the influence nanometer pinning centres 

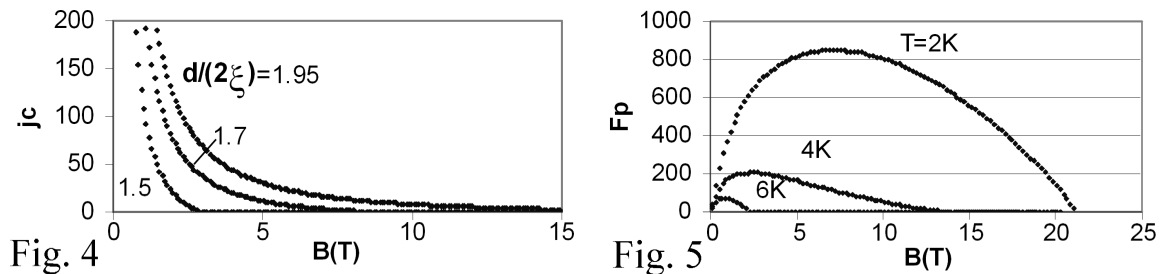

Fig. 4. The influence of the dimensions of nanoscale pinning centres on the critical current density as a function of magnetic field.

Fig. 5. The influence of the temperature on the magnetic field dependence of pinning forces $F_{\mathrm{p}}$ for nanoscale pinning centres.

on the critical current magnetic field dependence obtained in this model is shown in Fig. 4. From the above given local critical current values the averaged volume pinning force $F_{\mathrm{p}}$ has been calculated. At this aim the statistical occupation of the pinning centres by the vortices has been considered and the total pinning force determined. The results of calculations are presented in Fig. 5, which shows the temperature dependence of the volume pinning forces. This kinf of behaviour is in agreement with experimental data frequently presented in the literature.

\section{Acknowledgment}

The author is indebted to Prof. U. Hartmann and Dr. M. Koblischka from Saarbruecken University for hospitality.

\section{References}

[1] J. Sosnowski, M. Rabara, K. Demachi, K. Miya, Supercond. Sci. Technol. 13, 558 (2000).

[2] J. Sosnowski, Physica C 387, 239 (2003). 